Corresponding author: lesb@ mail.nih.gov

(C) 2017 Johnston et al. This article is distributed under the terms of the Creative Commons Attribution License, which permits unrestricted reuse and redistribution provided that the original author and source are credited.

Ontology terms: $2-3$ toe syndactyly; abnormality of the eyebrow; accessory oral frenulum; broad nasal tip; central hypotonia; cutaneous finger syndactyly; depressed nasal bridge; dysgenesis of the cerebellar vermis; high, narrow palate; hypertelorism; incomplete cleft of the upper lip; mixed hearing impairment; patent foramen ovale; postaxial hand polydactyly; preaxial foot polydactyly; prominent epicanthal folds; rhizomelic arm shortening; sparse scalp hair; thick anterior alveolar ridges; underdeveloped supraorbital ridges

Published by Cold Spring Harbor Laboratory Press

doi: 10.1101/mcs.a001321

\section{Compound heterozygous alterations in intraflagellar transport protein CLUAP1 in a child with a novel Joubert and oral-facial-digital overlap syndrome}

\author{
Jennifer J. Johnston, ${ }^{1,5}$ Chanjae Lee, ${ }^{2,5}$ Ingrid M. Wentzensen, ${ }^{1,5,6}$ \\ Melissa A. Parisi, ${ }^{3}$ Molly M. Crenshaw, ${ }^{1,7}$ Julie C. Sapp, ${ }^{1}$ Jeffrey M. Gross, ${ }^{4}$ \\ John B. Wallingford, ${ }^{2}$ and Leslie G. Biesecker ${ }^{1}$

\begin{abstract}
${ }^{1}$ Medical Genomics and Metabolic Genetics Branch, National Human Genome Research Institute, National of Texas at Austin, Austin, Texas 78705, USA; ${ }^{3}$ Intellectual and Developmental Disabilities Branch, Eunice Kennedy Shriver National Institute of Child Health and Human Development, National Institutes of Health, Bethesda, Maryland 20892, USA; ${ }^{4}$ Departments of Ophthalmology and Developmental Biology, University of Pittsburgh, Pittsburgh, Pennsylvania 15260, USA
\end{abstract} \\ Institutes of Health, Bethesda, Maryland 20892-4472, USA; ${ }^{2}$ Department of Molecular Biosciences, University
}

Abstract Disruption of normal ciliary function results in a range of diseases collectively referred to as ciliopathies. Here we report a child with a phenotype that overlapped with Joubert, oral-facial-digital, and Pallister-Hall syndromes including brain, limb, and craniofacial anomalies. We performed exome-sequence analysis on a proband and both parents, filtered for putative causative variants, and Sanger-verified variants of interest. Identified variants in CLUAP1 were functionally analyzed in a Xenopus system to determine their effect on ciliary function. Two variants in CLUAP1 were identified through exome-sequence analysis, Chr16:g.3558407T>G, c.338T>G, p.(Met113Arg) and Chr16: g.3570011C $>\mathrm{T}$, c.688C $>\mathrm{T}$, p.(Arg230Ter). These variants were rare in the Exome Aggregation Consortium (ExAC) data set of 65,000 individuals (one and two occurrences, respectively). Transfection of mutant CLUAP1 constructs into Xenopus embryos showed reduced protein levels p.(Arg230Ter) and reduced intraflagellar transport p.(Met113Arg). The genetic data show that these variants are present in an affected child, are rare in the population, and result in reduced, but not absent, intraflagellar transport. We conclude that biallelic mutations in CLUAP1 resulted in this novel ciliopathy syndrome in the proband.

\section{INTRODUCTION}

Human ciliopathies arise from mutation in any of dozens of genes implicated in ciliogenesis, and many of these genes are grouped in functional units. One such unit is formed by the intraflagellar transport (IFT) proteins, which collaborate to move cargo into and out of cilia

\footnotetext{
${ }^{5}$ These authors contributed equally to this work.

${ }^{6}$ Present address: GeneDx, Gaithersburg, Maryland 20877, USA

${ }^{7}$ Present address: University of North Carolina School of Medicine, Chapel Hill, North Carolina 27516, USA
} 
(Lechtreck 2015). Mutations in several IFT genes have been implicated previously in diverse ciliopathies (Beales et al. 2007; Arts et al. 2011; Boldt et al. 2011), as have proteins controlling recruitment and assembly of IFT particles (Toriyama et al. 2016). CLUAP1 is a relatively recently described member of the IFT complex, having been shown to be involved in ciliogenesis in mice, zebrafish, and the frog Xenopus (Pasek et al. 2012; Botilde et al. 2013; Lee et al. 2014). Recently, hypomorphic mutations were identified in human CLUAP1 associated with the retinal ciliopathy Leber congenital amaurosis (Soens et al. 2016).

Here, we expand the role of CLUAP1 in the ciliopathy spectrum by describing a proband originally enrolled in a study of the genetic etiology of polydactyly with additional associated features. Exome sequencing identified compound heterozygous mutations in CLUAP1, and functional analysis on the altered protein in the Xenopus model of intraflagellar transport demonstrates that these alleles disrupt the normal function of CLUAP1.

\section{RESULTS}

\section{Clinical Presentation and Family History}

The proband was born at 36 wk gestation, the second pregnancy of a 27 -yr-old female. On examination at $3 d$, he had sparse scalp hair and eyebrows, underdeveloped supraorbital ridges, apparently widely spaced eyes with epicanthal folds, a wide and mildly depressed nasal bridge, a broad nasal tip, and retrognathia. His oral findings included a midline notched upper lip, alveolar ridge overgrowth, high palate, extra frenula, a malformed epiglottis with a midline cleft, and a notched tongue tip (Fig. 1F). His limb findings were remarkable for mild rhizomelic shortening, bilateral postaxial polydactyly with partial cutaneous syndactyly of fingers 4-5, bilateral preaxial polydactyly with partial cutaneous syndactyly of toes 2-3, broadened metatarsals, short fingers and toes, and small nails (Fig. 1B-E). Additionally, a patent foramen ovale, breathing difficulties, and hypotonia were noted. $\mathrm{He}$ had mild bilateral mixed hearing loss, for which he had tympanostomy tubes and hearing aids placed at the age of $1 \mathrm{yr}$. At the age of $2 \mathrm{yr}$, magnetic resonance imaging (MRI) showed a mildly to moderately small cerebellar vermis, horizontal and thick superior cerebellar peduncles, superior cerebellar dysplasia, and cerebellar tonsil dysplasia, consistent with the molar tooth sign (Fig. 1A). Also at the age of $2 \mathrm{yr}$, a gastrostomy tube ( $\mathrm{G}$ tube) was placed for swallowing difficulty due to moderate pharyngeal phase dysphagia. At the age of $5 \mathrm{yr}$, a small penis and disproportionate rhizomelic shortening in the upper and lower limbs were noted. An electroencephalography for recurrent seizure episodes showed mild excessive background slowing for age; the episodes were described as petit mal or absence seizures possibly of frontotemporal origin. At this time, hypodontia, midline supernumerary tooth, fused teeth, narrow frenulum, a prominent upper lip, recurrent otitis media with effusion, chronic hearing loss likely mixed in nature, crowded oropharynx space (Mallampati type III), and tonsils 2-3+ in size were noted. At $9 \mathrm{yr}$, mild obstructive sleep apnea was diagnosed because of short stature, an increased body mass index (BMI) (see below for growth parameters), a small airway, redundant arytenoids, and hypotonia. At the age of $10 \mathrm{yr}$, he was noted to have a prominent epiglottis, a small larynx, and a bone age of 8-9 yr (mildly delayed). Growth parameters included an occipital-frontal circumference (OFC) of $55.2 \mathrm{~cm}$ (90th centile), height of $127.6 \mathrm{~cm}$ (2nd centile), weight of $45.6 \mathrm{~g}$ (92nd centile), and BMI of 28.0 (99th centile, obese). He had global developmental delay with oculomotor apraxia and drew pictures appropriate for a $4 \mathrm{yr}$ old. He had a speech delay with poor volume and articulation. His family history was unremarkable, and there was no consanguinity reported. Genetic testing was negative for MKS1, RPGRIP1L, AHI1, CEP290, TMEM67/MKS3, GLI3, NPHP1, CC2D2A, TMEM216, and OFD1. He was felt to have a phenotype that overlapped Joubert syndrome, oral-facial-digital syndromes (OFDSs), and Pallister-Hall syndrome (Table 1) (Hall et al. 1980; 

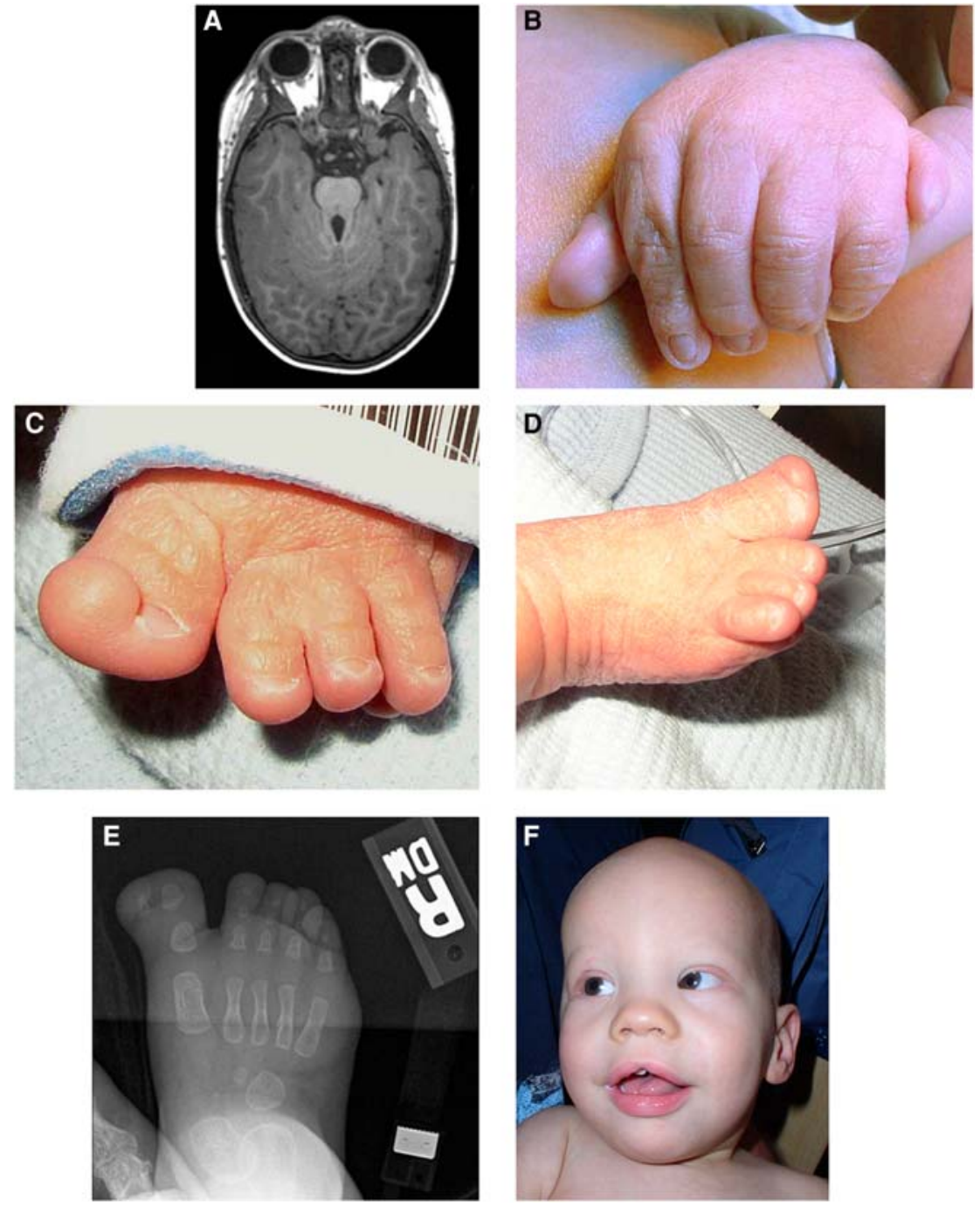

Figure 1. Patient images showing $(A)$ (via magnetic resonance imaging) the molar tooth sign; $(B)$ the left hand showing postaxial polydactyly and partial cutaneous syndactyly of the fourth and fifth digits; $(C)$ the left foot showing partial duplication of the hallux and partial cutaneous syndactyly of the second and third toes; $(D)$ the right foot showing the broad hallux and nail; $(E)$ (via radiograph) the right foot showing the duplicated distal phalanx of the hallux; and $(F)$ the proband as an infant showing sparse scalp hair and eyebrows, epicanthal folds, wide nasal bridge and tip, and midline notch in the upper lip.

Parisi and Glass 1993; Romani et al. 2013; Valente et al. 2013; Franco and Thauvin-Robinet 2016).

\section{Exome Analysis}

No variants passed our filtering criteria for either a de novo or autosomal recessive homozygous inheritance model. Variants were identified in two genes, LRRC4 and CLUAP1, which fit an autosomal recessive compound heterozygous model. LRRC4 is suggested to have a role in tumor progression (Zhang et al. 2005). Based on the role of CLUAP1 in ciliogenesis during embryonic development in model animals (Pasek et al. 2012; Botilde et al. 2013; Lee et al. 2014), we concluded that the variants in CLUAP1 were more likely to account for the 
Table 1. Clinical findings in the present patient compared with Joubert syndrome, oral-facial-digital syndrome (OFDS) type II, and PallisterHall syndrome

\begin{tabular}{|c|c|c|c|c|}
\hline & Proband & Joubert syndrome ${ }^{a}$ & OFDS type II & $\begin{array}{l}\text { Pallister-Hall } \\
\text { syndrome }\end{array}$ \\
\hline $\begin{array}{l}\text { Widely spaced eyes or dystopia } \\
\text { canthorum }\end{array}$ & + & $(+)$ & + & - \\
\hline Tongue clefts or hamartomas & Hamartomas & $\begin{array}{l}(+) \text { Clefts or } \\
\text { hamartomas }\end{array}$ & + Clefts & (+) Hamartomas \\
\hline Abnormal oral frenula & Numerous & $(+)$ & Numerous or thick & (+) Numerous \\
\hline Abnormal dentition & + & - & $(+)$ & - \\
\hline Midline cleft lip & + & $(+)$ & + & - \\
\hline Cleft palate & - & $(+)$ & $(+)$ & - \\
\hline Epiglottis bifid/cleft & + & - & - & + \\
\hline Polydactyly & $\begin{array}{l}\text { Postaxial hands and } \\
\text { preaxial of toes }\end{array}$ & $\begin{array}{l}(+) \text { Variable pre and } \\
\text { postaxial }\end{array}$ & $\begin{array}{l}\text { Postaxial hands and } \\
\text { preaxial of toes }\end{array}$ & $\begin{array}{l}\text { Mesoaxial or } \\
\text { postaxial }\end{array}$ \\
\hline Cutaneous syndactyly & $\begin{array}{l}\text { Involving duplicated } \\
\text { halluces }\end{array}$ & $(+)$ & $\begin{array}{l}\text { Involving duplicated } \\
\text { halluces }\end{array}$ & + \\
\hline Short fingers & - & - & $(+)$ & + \\
\hline Short limbs & + & - & $(+)$ & - \\
\hline Cystic/dysplastic kidneys & - & $(+)$ & - & $(+)$ \\
\hline Liver fibrosis & - & $(+)$ & - & - \\
\hline Retinal dystrophy & - & $(+)$ & - & - \\
\hline Leber congenital amaurosis & - & $(+)$ & - & - \\
\hline Intellectual disability & + & + & $(+)$ & - \\
\hline Hypotonia & + & + & $(+)$ & - \\
\hline Oculomotor apraxia & + & + & $(+)$ & - \\
\hline Breathing abnormalities & + & + & $(+)$ & - \\
\hline Molar tooth sign & + & + & - & - \\
\hline Encephalocele & - & $(+)$ & $(+)$ & - \\
\hline Polymicrogyria & - & $(+)$ & - & - \\
\hline Hypothalamic hamartoma & - & - & $(+)$ & + \\
\hline Porencephaly & - & - & $(+)$ & - \\
\hline Hydrocephalus & - & $(+)$ & $(+)$ & - \\
\hline
\end{tabular}

+, Typically present; (+) present but not consistent; -, not typically present.

a Joubert syndrome is genetically and clinically heterogeneous.

proband's phenotype. Variants, Chr16:g.3558407T>G c.338T>G, p.(Met113Arg) and Chr16:g.3570011C>T, c.688C >T, p.(Arg230Ter) (reference cDNA NM_015041.2) (Table 2), were Sanger-verified in the trio, with both variants present in the proband, c.338T $>C$ in the father and c.688C $>\mathrm{T}$ in the mother. Both variants were rare in the Exome Aggregation Consortium (ExAC; http://exac.broadinstitute.org) data set of 65,000 individuals (one and two occurrences, respectively) (Lek et al. 2016).

\section{Intraflagellar Transport and Localization Studies}

We used expression of green fluorescent protein (GFP)-tagged mutant CLUAP1 proteins in Xenopus embryos to assess the effect of the variants on localization and intraflagellar transport. The p.(Met113Arg) variant was localized normally to both the axoneme and the basal 


\begin{tabular}{|c|c|c|c|c|c|c|c|c|c|c|}
\hline Gene & Chrom & $\begin{array}{l}\text { DNA variant } \\
\text { hg19/GRC37 }\end{array}$ & $\begin{array}{l}\text { HGVS protein } \\
\text { Ref. }\end{array}$ & $\begin{array}{l}\text { Variant } \\
\text { type }\end{array}$ & $\begin{array}{l}\text { Predicted } \\
\text { effect }\end{array}$ & $\begin{array}{l}\text { dbSNP/ } \\
\text { dbVar ID }\end{array}$ & $\begin{array}{l}\text { Genotype } \\
\text { proband }\end{array}$ & $\begin{array}{c}\text { Genotype } \\
\text { mother }\end{array}$ & $\begin{array}{c}\text { Genotype } \\
\text { father }\end{array}$ & ClinVar ID \\
\hline \multirow[t]{2}{*}{ CLUAP1 } & 16 & $\begin{array}{l}\text { NC_000016.9: } \\
\text { g.3570011C>T }\end{array}$ & $\begin{array}{c}\text { XP_005255243.1: } \\
\text { p.Arg230Ter }\end{array}$ & $\begin{array}{l}\text { SNV; } \\
\quad \text { Nonsense }\end{array}$ & p.(Arg230Ter) & rs769705065 & CT & CT & $\mathrm{CC}$ & SCV000299272 \\
\hline & & & & & & Coverage: & 26 & 28 & 35 & \\
\hline \multirow[t]{2}{*}{ CLUAP1 } & 16 & $\begin{array}{l}\text { NC_000016.9: } \\
\text { g.3558407T>G }\end{array}$ & $\begin{array}{l}\text { XP_005255243.1: } \\
\text { p.Met113Arg }\end{array}$ & $\begin{array}{l}\text { SNV; } \\
\quad \text { Missense }\end{array}$ & p.(Met113Arg) & ) rs768663992 & GT & TT & GT & SCV000299273 \\
\hline & & & & & & Coverage: & 28 & 40 & 31 & \\
\hline
\end{tabular}

HGVS, Human Genome Variation Society; dbSNP, Database for Short Genetic Variations; dbVar, Database of Genomic Structural Variation; SNV, single-nucleotide variant.

body (Fig. 2A,B,D,E). In contrast, the nonsense mutation, p.(Arg230Ter), resulted in extremely low protein levels (Fig. 2C,F), a result that was confirmed by western blotting (Fig. 2G), so no further assessment was possible. Western blotting revealed that p.(Met113Arg) was expressed at levels comparable to wild-type Cluap1 in Xenopus embryos.

Because the p.(Met113Arg) variant was expressed and localized normally, we turned to high-speed confocal imaging of IFT movements to determine whether this variant had a more subtle defect. Indeed, we found that the average velocity for both anterograde and retrograde transport were reduced for the mutant when compared with wild type (Fig. 3). Though relatively minor, these reductions in IFT rate were comparable to those associated with defective ciliogenesis in previous studies (Tran et al. 2008; Chung et al. 2014). Together, our data suggest that the p.(Arg230Ter) truncation is likely a null allele, whereas the p.(Met113Arg) is likely hypomorphic.

\section{DISCUSSION}

Disorders of ciliary function are collectively known as ciliopathies and include diseases that affect single organ systems to multisystemic developmental disorders including polycystic kidney disease, retinal dystrophy, Meckel syndrome, Joubert syndrome, OFDS, and others (Ferkol and Leigh 2012). IFT, the active movement of proteins within the cilium, plays a primary role in the formation of the cilia and cilia-mediated signaling. Disruption of this process through mutations in a number of genes that code for ciliary components causes ciliopathies (Lechtreck 2015). CLUAP1 has been identified as a component of the IFT complex B and is believed to regulate the IFT cycle at the base and tip of the cilium. Recently, a single proband has been described with Leber congenital amaurosis with a homozygous missense mutation in CLUAP1, c.817C > T; p.(Leu273Phe), supporting the role of CLUAP1 in ciliary function (Soens et al. 2016). Here we report the identification of compound heterozygous mutations in CLUAP1 in a proband with a phenotype that included limb, central nervous system (CNS), and craniofacial anomalies that overlapped with Joubert syndrome, OFDS, and Pallister-Hall syndrome, thus expanding the understanding of CLUAP1 in disease.

The implication of CLUAP1 in ciliopathies is supported by diverse data from model animals. For example, zebrafish au5 mutants demonstrate photoreceptor degeneration, embryonic lethality, and defects of ciliogenesis (Lee et al. 2014) owing to a nonsense mutation in cluap1. Likewise, knockout of Cluap1 in mice leads to embryonic lethality with abnormal heart looping and embryonic turning (Botilde et al. 2013), consistent with early defects in ciliogenesis. The proband presented here was compound heterozygous for a nonsense, 

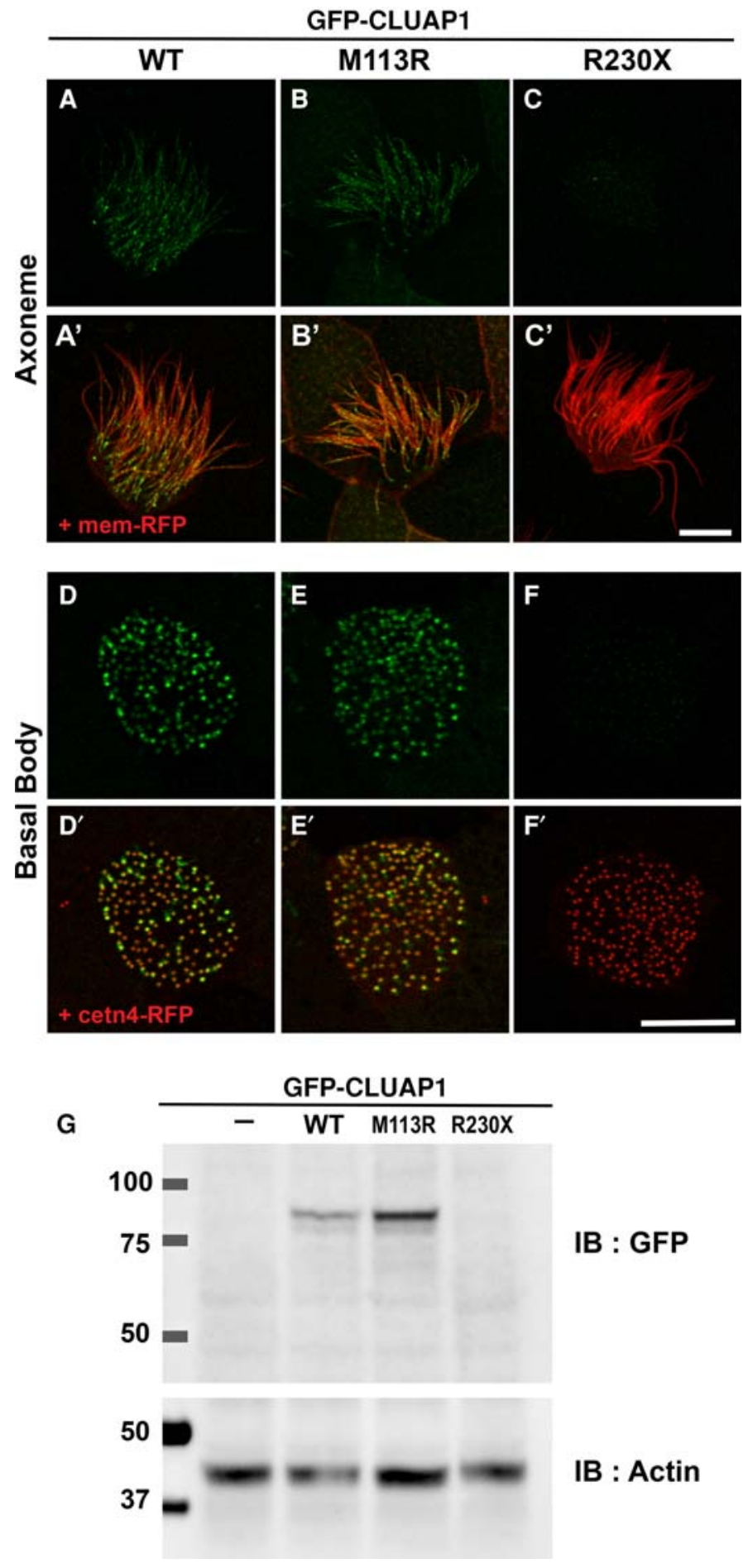

Figure 2. The CLUAP1 M113R mutant is expressed and localized normally, whereas the expression level of R230X mutants is extremely low. (A-F) Live images displaying localization of green fluorescent protein (GFP)-tagged CLUAP1 WT, M113R, and R230X proteins at the axonemes (A-C) and basal bodies (D-H) in Xenopus multiciliated cells. $\left(A^{\prime}-C^{\prime}\right)$ Merge views with membrane red fluorescent protein (RFP) visualizing axonemes. $\left(D^{\prime}-F^{\prime}\right)$ Merge views with cetn4-RFP, a basal body marker. Scale bars, $10 \mu \mathrm{m}$. (G) Western blotting of GFP-tagged CLUAP1 proteins. Actin was used as a loading control. 


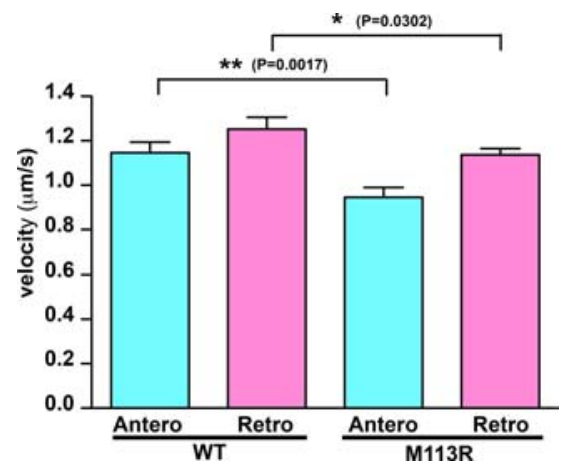

Figure 3. The CLUAP1 M113R mutant has significantly reduced intraflagellar transport (IFT) velocities. The graph shows velocities of green fluorescent protein (GFP)-tagged CLUAP1 wild type (WT) and M113R. Mean velocities \pm SEM of CLUAP1 WT are $1.15 \pm 0.05 \mu \mathrm{m} / \mathrm{sec}$ for anterograde $(N=33)$ and $1.25 \pm 0.05 \mu \mathrm{m} /$ sec for retrograde $(N=61)$. Mean velocities \pm SEM of CLUAP1 M113R are $0.95 \pm 0.05 \mu \mathrm{m} / \mathrm{sec}$ for anterograde $(N=42)$ and $1.14 \pm 0.03 \mu \mathrm{m} / \mathrm{sec}$ for retrograde $(N=76)$. Comparing with $W T$, IFT velocities of M113R are significantly reduced ( $P$ value of WT vs. M113R is 0.0017 for anterograde and 0.0302 for retrograde).

p.(Arg230Ter), and a missense, p.(Met113Arg), mutation in CLUAP1. The nonsense mutation should result in limited protein production, presumably because of nonsense-mediated decay, whereas the missense mutation is expected to be hypomorphic, allowing for sufficient CLUAP1 function to escape embryonic lethality. Indeed, the p.(Met113Arg) mutation produced similar levels of protein when compared with wild type, but this p.(Met113Arg) mutant demonstrated slower IFT transport when compared with wild-type protein. TTC21B is another ciliary gene, mutations in which have been shown to cause nephronophthisis type 12 (OMIM [Online Mendelian Inheritance in Man; http://www.omim.org/] 613820) and shortrib thoracic dysplasia type 4 (OMIM 613819) (Davis et al. 2011). Slow IFT transport has been reported for Ttc21b mutant mice with concomitant dysregulation of the Sonic hedgehog (SHH) pathway (Tran et al. 2008), supporting a causal relationship of IFT slowing and the phenotype in the present patient.

The patient with CLUAP1 mutations and Leber congenital amaurosis had severe visual impairment with vision limited to light perception by 6 wk of age without other systemic features (Soens et al. 2016), whereas the patient presented here has a pleiotropic developmental disorder with features including polydactyly, cutaneous syndactyly, oculomotor apraxia, respiratory dysfunction, obesity, and an abnormal cerebellar vermis. When last seen at $9 \mathrm{yr}$ of age, he did not have retinal degeneration. It is likely that the effects on protein function of the missense mutations identified in these two individuals are distinct, leading to the different phenotypes. The p.(Leu273Phe) variant in the patient with Leber congenital amaurosis was in the coiled-coil domain; amino acids 187-308 of CLUAP1 are known to be important in homopolymer formation. The p.(Met113Arg) mutation reported here is located within the calponin homology domain of CLUAP1, which is known to be important for interaction with another IFT protein, IFT80 (Taschner et al. 2016), also implicated in ciliopathy disorders. Alternatively, the variable phenotypes seen in these two patients could be related to the effects of other unknown modifying genes.

We conclude that the CLUAP1 variants identified here are pathogenic and cause a phenotype within the Joubert syndrome spectrum. This is based on the finding of the molar tooth sign, considered pathognomonic for Joubert syndrome, the previously demonstrated role of CLUAP1 variants in Leber congenital amaurosis (a known ciliopathy), biallelic variants that are rare but are predicted to be pathogenic, and functional analysis showing decreased IFT in a validated Xenopus model system. Mutation scanning for patients with Joubert 
syndrome and related phenotypes should include CLUAP1 as a candidate gene. The identification of additional affected patients is essential to identify the mutational and phenotypic heterogeneity of this disorder.

\section{METHODS}

\section{Exome Sequencing}

DNA was isolated from the proband and his parents' whole blood using the salting-out method (QIAGEN), followed by phenol-chloroform extraction. Solution hybridization exome capture was performed using the Illumina TruSeq system (Illumina). Flow cell preparation and paired-end read sequencing were performed using the HiSeq 2000 sequencer (Vilboux et al. 2016) (Illumina). Image analyses and base-calling were performed as described (Vilboux et al. 2016). Reads were aligned to hg19, NCBI 37, using NovoAlign (Novocraft Technologies). Samples were sequenced to sufficient coverage such that at least $85 \%$ of the targeted exome was called with high-quality variant detection (reported as genotype at every callable position). Genotypes were called using only those sequence bases with Phred base qualities of at least Q20 using Most Probable Genotype (Teer et al. 2010) (MPG) and an MPG score of $\geq 10$. Filters were applied using the VarSifter Next-Gen variant analysis software (Teer et al. 2012). Variants were filtered for nonsynonymous, splice-site, frameshift, and nonsense alterations. To filter for rare variants, the ClinSeq cohort (1001 control individuals) was used with an initial minor allele frequency (MAF) filter of $<0.005$. Results were analyzed using both recessive and dominant de novo models. Sanger sequencing was performed for confirmation of variants detected by exome sequencing. Primer sequences included CLUAP1_1F, ATC CCA GTT GTC AGC AGA GG; CLUAP1_1R, CCA AAT GCT GAT GAG CAC AA; CLUAP1_2F, TGC TTG GGG AAG TGT CTA TTT; CLUAP1_2R, TTT CAG ACA TGA GCC ACC AC. Identified variants were assessed in the ExAC data set of 65,000 individuals to assess their population frequency (Lek et al. 2016). Mutation nomenclature is according to Human Genome Variation Society (HGVS) standards using reference sequence NM_015041.2.

\section{Plasmids and Microinjections to Xenopus Embryos}

The wild-type CLUAP1 open reading frame was cloned from human CDNA and inserted into pCS10R-GFP vector. To generate GFP-tagged mutant CLUAP1 constructs, mutagenesis was performed with a QuikChange II site-Direct Mutagenesis Kit (Agilent Technologies). Capped and polyadenylated mRNAs were synthesized via an mMESSAGE mMACHINE SP6 Transcription Kit (Life Technologies). Of note, 100 or 150 pg of GFP-CLUAP1 mRNA, 50 pg of cent4-RFP mRNA, and 50 pg mem-RFP mRNA were injected into two ventral blastomeres of four-cell stage embryos.

\section{Live Imaging and Analysis}

Embryos at stage $25-28$ were anesthetized with $0.005 \%$ benzocaine. Images were captured with a LSM 700 confocal microscope with an $63 \times / 1.4$ oil immersion objective (Carl Zeiss). High-speed in vivo images were acquired on a Nikon Eclipse Ti confocal microscope with a $63 \times / 1.4$ oil immersion objective. Time-lapse series were captured $266.5 \mathrm{msec}$ per frame. Images were analyzed by ImageJ.

\section{Western Blotting}

Embryos were lysed in M-PERTM Mammalian Protein Extraction Reagent (ThermoFisher Scientific) containing protease inhibitor cocktail (Sigma-Aldrich) and phenylmethylsulfonyl 
Competing Interest Statement L.G.B. is an uncompensated advisor to the Illumina Corp. and receives honoraria from WileyBlackwell. The other authors declare no competing interests.

\section{Referees}

Peter N. Robinson

Anonymous

Received August 4, 2016; accepted in revised form October 5, 2016. fluoride. Embryo extracts were applied to sodium dodecyl sulfate (SDS)-polyacrylamide gel electrophoresis (PAGE) gels and transferred onto nitrocellulose membrane. The GFP (1:2000 dilution, sc-9996, Santa Cruz) and actin (1:2000 dilution, ab1801, Abcam) antibodies were used as primary antibodies. The GFP or actin proteins were detected with horseradish peroxidase (HRP)-anti mouse or rabbit IgG antibody (1:10,000, Pierce or Jackson ImmunoResearch Laboratories, respectively) and visualized with the SuperSignal West Dura Extended Duration Substrate (Pierce).

\section{ADDITIONAL INFORMATION}

\section{Data Deposition and Access}

The sequencing data is available in the National Center for Biotechnology Information (NCBI) database of Genotypes and Phenotypes (dbGaP; https://www.ncbi.nlm.nih.gov/ gap) under study number phs001348.v1.p1. Variants have been submitted to ClinVar (https://www.ncbi.nlm.nih.gov/clinvar/) under accession numbers SCV000299272 and SCV000299273.

\section{Ethics Statement}

This study was performed under National Human Genome Research Institute (NHGRI) institutional review board (IRB)-approved protocols 10-HG-0065 and 94-HG-01903 that include approval for publication of results. Written informed consent was obtained according to those approved protocols.

\section{Acknowledgments}

The authors gratefully acknowledge the family for their contributions to this work.

\section{Author Contributions}

L.G.B., J.B.W., and J.M.G. designed and interpreted the studies. J.J.J. and I.M.W. performed and interpreted the human genomic analyses; C.L. performed and interpreted the animal model experiments. M.A.P., M.M.C., and J.C.S. performed the clinical analyses. J.J.J. wrote the initial draft of the manuscript, and all coauthors edited it for important scientific content.

\section{Funding}

This work was supported by funding from the Intramural Research Program of the National Human Genome Research Institute (1 ZIA HG200328 11 and 1 ZIA HG200388 03 to L.G.B., J.J.J., M.M.C., J.C.S., and I.M.W.). C.L. and J.B.W. were supported by funding from the NHLBI (HL117164).

\section{REFERENCES}

Arts $\mathrm{HH}$, Bongers EM, Mans DA, van Beersum SE, Oud MM, Bolat E, Spruijt L, Cornelissen EA, SchuursHoeijmakers JH, de Leeuw N, et al. 2011. C14ORF179 encoding IFT43 is mutated in Sensenbrenner syndrome. J Med Genet 48: 390-395.

Beales PL, Bland E, Tobin JL, Bacchelli C, Tuysuz B, Hill J, Rix S, Pearson CG, Kai M, Hartley J, et al. 2007. IFT80, which encodes a conserved intraflagellar transport protein, is mutated in Jeune asphyxiating thoracic dystrophy. Nat Genet 39: 727-729. 
Boldt K, Mans DA, Won J, van Reeuwijk J, Vogt A, KinkI N, Letteboer SJ, Hicks WL, Hurd RE, Naggert JK, et al. 2011. Disruption of intraflagellar protein transport in photoreceptor cilia causes Leber congenital amaurosis in humans and mice. J Clin Invest 121: 2169-2180.

Botilde Y, Yoshiba S, Shinohara K, Hasegawa T, Nishimura H, Shiratori H, Hamada H. 2013. Cluap1 localizes preferentially to the base and tip of cilia and is required for ciliogenesis in the mouse embryo. Dev Bio/ 381: 203-212.

Chung MI, Kwon T, Tu F, Brooks ER, Gupta R, Meyer M, Baker JC, Marcotte EM, Wallingford JB. 2014. Coordinated genomic control of ciliogenesis and cell movement by RFX2. Elife 3: e01439.

Davis EE, Zhang Q, Liu Q, Diplas BH, Davey LM, Hartley J, Stoetzel C, Szymanska K, Ramaswami G, Logan CV, et al. 2011. TTC21B contributes both causal and modifying alleles across the ciliopathy spectrum. Nat Genet 43: 189-196.

Ferkol TW, Leigh MW. 2012. Ciliopathies: the central role of cilia in a spectrum of pediatric disorders. J Pediatr 160: 366-371.

Franco B, Thauvin-Robinet C. 2016. Update on oral-facial-digital syndromes (OFDS). Cilia 5: 12.

Hall JG, Pallister PD, Clarren SK, Beckwith JB, Wiglesworth FW, Fraser FC, Cho S, Benke PJ, Reed SD. 1980. Congenital hypothalamic hamartoblastoma, hypopituitarism, imperforate anus and postaxial polydactyly - a new syndrome? Part I: clinical, causal, and pathogenetic considerations. Am J Med Genet 7: 47-74.

Lechtreck KF. 2015. IFT-cargo interactions and protein transport in cilia. Trends Biochem Sci 40: 765-778.

Lee C, Wallingford JB, Gross JM. 2014. Cluap1 is essential for ciliogenesis and photoreceptor maintenance in the vertebrate eye. Invest Ophthalmol Vis Sci 55: 4585-4592.

Lek M, Karczewski KJ, Minikel EV, Samocha KE, Banks E, Fennell T, O’Donnell-Luria AH, Ware JS, Hill AJ, Cummings BB, et al. 2016. Analysis of protein-coding genetic variation in 60,706 humans. Nature 536: 285-291.

Parisi M, Glass I. 1993. Joubert syndrome and related disorders. In GeneReviews (ed. Pagon RA, et al.). University of Washington, Seattle, WA.

Pasek RC, Berbari NF, Lewis WR, Kesterson RA, Yoder BK. 2012. Mammalian Clusterin associated protein 1 is an evolutionarily conserved protein required for ciliogenesis. Cilia 1: 20.

Romani M, Micalizzi A, Valente EM. 2013. Joubert syndrome: congenital cerebellar ataxia with the molar tooth. Lancet Neurol 12: 894-905.

Soens ZT, Li Y, Zhao L, Eblimit A, Dharmat R, Li Y, Chen Y, Naqeeb M, Fajardo N, Lopez I, et al. 2016. Hypomorphic mutations identified in the candidate Leber congenital amaurosis gene CLUAP1. Genet Med 18: 1044-1051.

Taschner M, Weber K, Mourao A, Vetter M, Awasthi M, Stiegler M, Bhogaraju S, Lorentzen E. 2016. Intraflagellar transport proteins $172,80,57,54,38$, and 20 form a stable tubulin-binding IFT-B2 complex. EMBO J 35: 773-790.

Teer JK, Bonnycastle LL, Chines PS, Hansen NF, Aoyama N, Swift AJ, Abaan HO, Albert TJ, Margulies EH, Green ED, et al. 2010. Systematic comparison of three genomic enrichment methods for massively parallel DNA sequencing. Genome Res 20: 1420-1431.

Teer JK, Green ED, Mullikin JC, Biesecker LG. 2012. VarSifter: visualizing and analyzing exome-scale sequence variation data on a desktop computer. Bioinformatics 28: 599-600.

Toriyama M, Lee C, Taylor SP, Duran I, Cohn DH, Bruel AL, Tabler JM, Drew K, Kelly MR, Kim S, et al. 2016. The ciliopathy-associated CPLANE proteins direct basal body recruitment of intraflagellar transport machinery. Nat Genet 48: 648-656.

Tran PV, Haycraft CJ, Besschetnova TY, Turbe-Doan A, Stottmann RW, Herron BJ, Chesebro AL, Qiu H, Scherz PJ, Shah JV, et al. 2008. THM1 negatively modulates mouse sonic hedgehog signal transduction and affects retrograde intraflagellar transport in cilia. Nat Genet 40: 403-410.

Valente EM, Dallapiccola B, Bertini E. 2013. Joubert syndrome and related disorders. Handb Clin Neurol 113: 1879-1888.

Vilboux T, Malicdan MC, Chang YM, Guo J, Zerfas PM, Stephen J, Cullinane AR, Bryant J, Fischer R, Brooks BP, et al. 2016. Cystic cerebellar dysplasia and biallelic LAMA1 mutations: a lamininopathy associated with tics, obsessive compulsive traits and myopia due to cell adhesion and migration defects. J Med Genet 53: 318-329.

Zhang Q, Wang J, Fan S, Wang L, Cao L, Tang K, Peng C, Li Z, Li W, Gan K, et al. 2005. Expression and functional characterization of LRRC4, a novel brain-specific member of the LRR superfamily. FEBS Lett $\mathbf{5 7 9}$ 3674-3682. 


\section{COLD SPRING HARBOR Molecular Case Studies}

\section{Compound heterozygous alterations in intraflagellar transport protein CLUAP1 in a child with a novel Joubert and oral-facial- digital overlap syndrome}

Jennifer J. Johnston, Chanjae Lee, Ingrid M. Wentzensen, et al.

Cold Spring Harb Mol Case Stud 2017, 3: a001321 originally published online April 12, 2017

Access the most recent version at doi: $10.1101 / \mathrm{mcs} .0001321$

References This article cites 23 articles, 5 of which can be accessed free at:

http://molecularcasestudies.cshlp.org/content/3/4/a001321.full.html\#ref-list-1

License This article is distributed under the terms of the Creative Commons Attribution License, which permits unrestricted reuse and redistribution provided that the original author and source are credited.

Email Alerting Receive free email alerts when new articles cite this article - sign up in the box at the Service top right corner of the article or click here. 